\title{
The chlorosome: a prototype for efficient light harvesting in photosynthesis
}

\author{
Gert T. Oostergetel $\cdot$ Herbert van Amerongen • \\ Egbert J. Boekema
}

Received: 24 November 2009/ Accepted: 16 January 2010/Published online: 4 February 2010

(C) The Author(s) 2010. This article is published with open access at Springerlink.com

\begin{abstract}
Three phyla of bacteria include phototrophs that contain unique antenna systems, chlorosomes, as the principal light-harvesting apparatus. Chlorosomes are the largest known supramolecular antenna systems and contain hundreds of thousands of BChl $c / d / e$ molecules enclosed by a single membrane leaflet and a baseplate. The BChl pigments are organized via self-assembly and do not require proteins to provide a scaffold for efficient light harvesting. Their excitation energy flows via a small protein, CsmA embedded in the baseplate to the photosynthetic reaction centres. Chlorosomes allow for photosynthesis at very low light intensities by ultra-rapid transfer of excitations to reaction centres and enable organisms with chlorosomes to live at extraordinarily low light intensities under which no other phototrophic organisms can grow. This article reviews several aspects of chlorosomes: the supramolecular and molecular organizations and the light-harvesting and spectroscopic properties. In addition, it provides some novel information about the organization of the baseplate.
\end{abstract}

Keywords Chlorosome - Photosynthesis · Electron microscopy · Spectroscopy

G. T. Oostergetel · E. J. Boekema ( $₫)$

Biophysical Chemistry, Groningen Biomolecular Sciences

\& Biotechnology Institute, University of Groningen,

Nijenborgh 4, 9747 AG Groningen, The Netherlands

e-mail: e.j.boekema@rug.nl

H. van Amerongen

Laboratory of Biophysics, Wageningen University,

P.O. Box 8128, 6700 ET Wageningen, The Netherlands

\section{Introduction}

Since the earliest photosynthetic organisms developed reaction centres, additional peripheral antenna systems have evolved for light harvesting. In these light-harvesting systems, dozens, hundreds or even thousands of (bacterio)chlorophylls can funnel their excitation energy towards reaction centres for charge separation. The green photosynthetic bacteria are anoxygenic phototrophs that contain unique antenna complexes, known as chlorosomes (Blankenship and Matsuura 2003). A chlorosome is actually a kind of organelle. In addition to the green sulphur bacteria (phylum Chlorobi), they are also present in some filamentous anoxygenic phototrophs of the phylum Chloroflexi (formerly know as green non-sulphur bacteria), and in the newly discovered aerobic phototroph, Candidatus Chloracidobacterium thermophilum (Cab. thermophilum) of the phylum Acidobacteria (Bryant et al. 2007). The green sulphur bacteria form the best studied group, and especially Chlorobaculum tepidum (also known as Chlorobium) from the family of Chlorobiaceae, has emerged as a model organism for the group. Within these organisms, the flow of excitation energy goes in the following direction:

Pigments within chlorosomes

$\rightarrow$ CsmA protein in baseplate $\rightarrow$ FMO protein

$\rightarrow$ reaction center.

Before discussing the structure and function of chlorosomes, some basic facts about the reaction centre and attached proteins are provided. The green sulphur bacterial reaction centre $(\mathrm{RC})$ is a multi-subunit complex, consisting of two copies of the PscA subunit of $82 \mathrm{kDa}$ protein which forms a homodimer, a single copy of PscB (23 kDa), two copies of a 23-kDa subunit named PscC, which in most recent papers is referred to as C-cyt ${ }_{c z}$ 
(Higuchi et al. 2009) and two copies of PscD of $15 \mathrm{kDa}$ (Hauska et al. 2001). The PscA subunit shows some sequence similarity to the heterodimer-forming subunits PsaA and PsaB of photosystem I of green plants and cyanobacteria. The RC shares also other similarities to those of heliobacteria and photosystem I. These include the presence of the same set of electron acceptors (Hauska et al. 2001; Oh-oka 2007). The RC is also similar to the purple bacterial type RC in the way that a bacteriochlorophyll $a$ dimer (P840) serves as the primary donor. Furthermore, the cytochrome protein $\mathrm{C}-\mathrm{cyt}_{\mathrm{cz}}$ acts as electron donor to the photo-oxidized P840. In the Chlorobiaceae species, the Ccyt $_{\mathrm{cz}}$ consists of an $\mathrm{N}$-terminal transmembrane domain and a C-terminal soluble domain that binds the single heme group. The small PscB protein binds two [4Fe-4S] clusters and is thus involved in electron transfer, in a similar way that PsaC is functioning in the cyanobacterial photosystem I protein complex. The PscD protein may be involved in stabilization of PscB and/or in the interaction with ferredoxin (see Hauska et al. 2001).

Two copies of the FMO protein trimer associate with the RC and electron microscopy analysis indicated that they are located close to the PscB and PscD subunits (see Hauska et al. 2001). The protein consists of three identical subunits, each with a mass of $40 \mathrm{kDa}$, and the structure of the FMO protein from two species has been determined by X-ray crystallography. The structures of Prosthecochloris aestuarii (Fenna and Matthews 1975; Tronrud et al. 1986) and Chlorobaculum tepidum (Li et al. 1997) show strong structural similarities. The three monomers form a disc with the symmetry axis perpendicular to the disc plane. There are seven BChl $a$ molecules in a cluster per monomer, and an eighth Bchl $a$ molecule has been resolved in newly solved structures. Recent chemical labelling and mass spectrometry data have established the orientation of the FMO protein on the membrane (Wen et al. 2008). This gave insight in the position of the BChls and how these pigments bridge the distance between the baseplate pigments and the core Bchl $a$ molecules in the FMO, and how they are involved in efficient excitation energy transfer (Tronrud et al. 2009).

\section{Chlorosomes}

Chlorosomes are the largest known antenna structures with some hundreds thousands of bacteriochlorophyll (BChl) $c$-, $d$ - or $e$-molecules per chlorosome, which means that there are at least some 5,000 BChls per RC (Hauska et al. 2001). A single chlorosome can contain, depending on the species, about 200,000 to 250,000 BChl $c$ and BChl $d$ molecules, 2,500 BChl a molecules, 20,000 carotenoid molecules, 15,000 chlorobiumquinone molecules, 3,000 menaquinone-7 molecules, 5,000 protein molecules of 10 different types, and about 20,000 lipid molecules (Bryant and Frigaard 2006). Chlorosomes efficiently capture light and this allows organisms that use chlorosomes for light harvesting to live at extraordinarily low light intensities under which no other phototrophic organisms can grow, exemplified by the findings of species able to survive $100 \mathrm{~m}$ below the surface of the Black Sea (Manske et al. 2005).

An interesting property of the chlorosomes is the fact that the majority of the pigments is organized via self-assembly and does not require proteins to provide a scaffold for efficient light harvesting, like the light-harvesting proteins in green plants. This is the major reason why chlorosomes form a source of inspiration for the design of artificial lightharvesting systems. (For a comprehensive review for the self-assembly of chlorins, see Balaban et al. 2005.) In this article, we will review the structural components involved in light harvesting in chlorosomes and their organization. The spectroscopic properties will also be discussed, in relation to the functioning of the chlorosomes and also in relation to the consequences for the structural organization, which after all is still not exactly known.

\section{Supramolecular organization of chlorophylls}

Chlorosomes can be considered as elongated sacks, 100$200 \mathrm{~nm}$ in length and 40-60 nm in diameter. The overall shape and size of isolated chlorosomes can be easily studied with transmission electron microscopy by classical negative staining with uranyl acetate (Fig. 1). This shows that chlorosomes from different species can differ by at least a factor of 5 in their volume and also vary in shape (Fig. 1,2). Some are ellipsoid shaped (Fig. 1a), whereas other are conically shaped (Fig. 1b) or irregularly shaped (Fig. 1c). Negative staining has, however, one drawback because it enhances only the contrast of the water-accessible surface; the small negative stain clusters do not penetrate the hydrophobic interior. Cryo-electron microscopy (cryo-EM) of frozen-hydrated samples, on the other hand, gives a total projected density, including the BChl structures. Chlorosomes of C. tepidum, embedded in an amorphous ice layer, give hints of the overall and internal structure. In unstained chlorosomes, a striation pattern is revealed, in a direction parallel to the long axis (Fig. 2a); its calculated diffraction pattern indicates a strong diffraction spot equivalent with a 2.1-nm spacing (inset, Fig. 2a).

Early observations by Staehelin and colleagues indicated that the chlorosome core is separated from the cytoplasm by an approx. 3-nm thick lipid-like envelope layer, which exhibits no substructure (Staehelin et al. 1980). The thickness of the surface layer-the chlorosome envelope-suggests that chlorosomes are surrounded by a lipid monolayer. Since then no further investigations have 

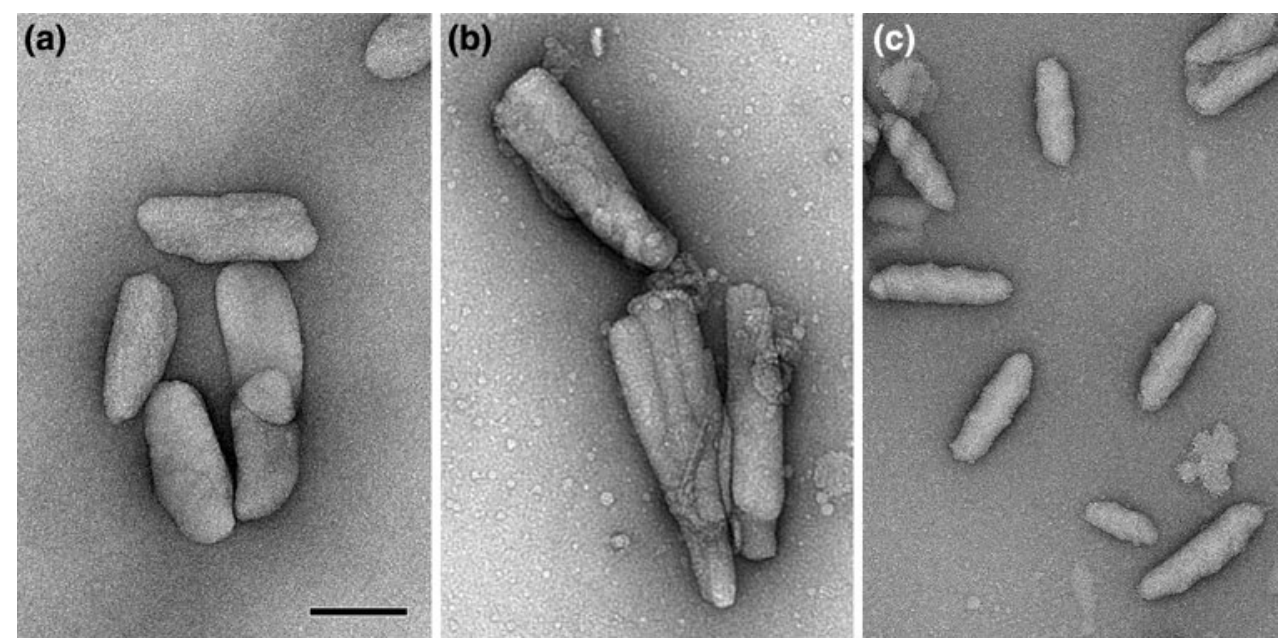

Fig. 1 Examples of isolated chlorosomes differing in overall shape and size. Specimens were prepared by negative stain embedding with uranyl acetate. a Ellipsoid-shaped chlorosomes of Chlorobaculum tepidum wild-type, the model organism of the green sulphur bacteria. b Conically shaped chlorosomes of Chlorobaculum tepidum
bchQRU mutant. c Irregularly shaped chlorosomes with a somewhat undulating surface of Cab. thermophilum, a newly discovered phototrophic microorganism belonging to the Acidobacteria. Size bar for all frames equals $100 \mathrm{~nm}$

layer of vitreous ice to reveal the arrangement of $\mathrm{BChl}$ sheets in wild-type chlorosomes and in chlorosomes from the triple mutant bchQRU (Gomez Maqueo Chew et al. 2007), which contains a well-defined $>95 \%$ homogeneous BChl $d$ (Oostergetel et al. 2007). End-on views of chlorosomes fixed in a vertical position gave a direct clue to the packing of the sheets. They show the presence of multilamellar tubules of variable diameter $(10-30 \mathrm{~nm})$ with some non-tubular locally curved lamellae in between (Fig. 3). In the bchQRU mutant, most chlorosomes contain two tubular domains, as can be deduced from the banding pattern of the 2-nm striations. Overall, the cryo-electron microscopy data show that the C. tepidum chlorosomes comprise multi-lamellar tubular domains extending over most of the length of the chlorosome, embedded in a less well-ordered matrix of smaller curved lamellar domains. The notion of multi-walled cylinders is consistent with the results from both freeze-fracture experiments done several decades ago and the more recent cryo-EM observations.

\section{Molecular organization of chlorophylls}

In addition to the 2-nm lamellar structure, cryo-EM images of C. tepidum chlorosomes and their calculated diffraction patterns indicated the presence of a smaller spaced regular structure in the direction of the long axis (Fig. 4). In wildtype chlorosomes, a weak periodicity of $1.25 \mathrm{~nm}$ is present (red arrow in Fig. 4b), in the bchQRU mutant a relatively strong $0.83 \mathrm{~nm}$ regular structure is evident from the diffraction pattern (Fig. 4d) and also directly visible in the image (Fig. 4c, inset). These cryo-EM observations 

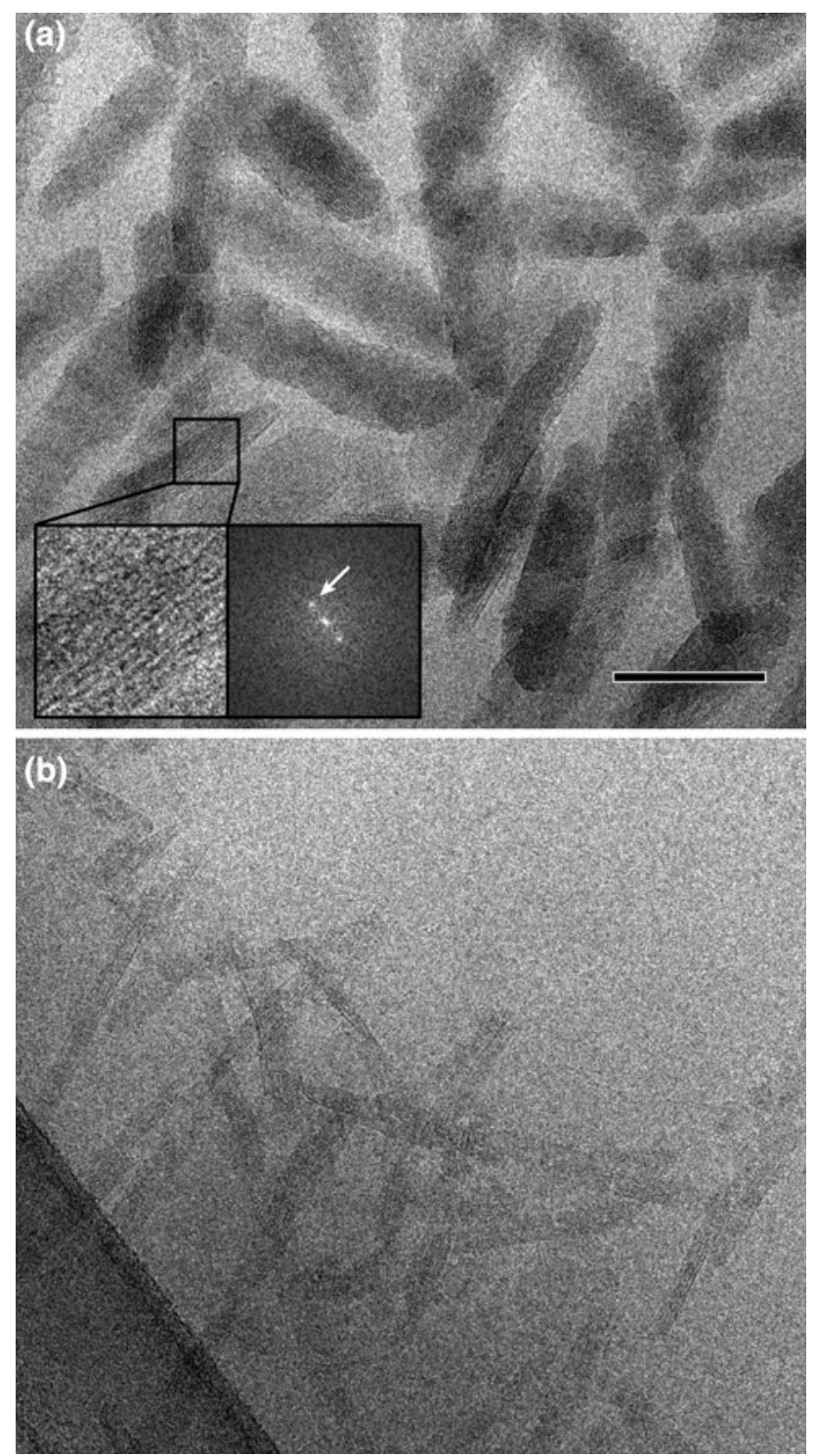

Fig. 2 Isolated chlorosomes embedded in an amorphous ice layer give hints of the overall and internal structure. a Overview of unstained chlorosomes of Chlorobium tepidum. The inset shows a fine parallel spacing of lamellae, its calculated diffraction pattern indicates a strong diffraction spot equivalent with a 2.1-nm lamellar spacing. b Unstained ice-embedded chlorosomes of Chloroflexus aurantiacus (phylum Chloroflexi or filamentous anoxygenic phototrophs). The ice layer has been prepared over a holey-carbon film, which is visible at the lower left side. Size bar for both frames equals $100 \mathrm{~nm}$

provide constraints concerning possible packing modes of the BChl molecules in the multi-lamellar tubes.

Due to absence of side chain heterogeneity at C-8 and $\mathrm{C}-12$, limited stereochemical heterogeneity at $\mathrm{C}-3^{1}$ and absence of a methyl group at C-20 in the bchQRU mutant very high resolution magic-angle-spinning (MAS) solidstate NMR data could be obtained. An alternating syn-antiligated BChl $d$ stack (Fig. 5a) and an antiparallel monomer
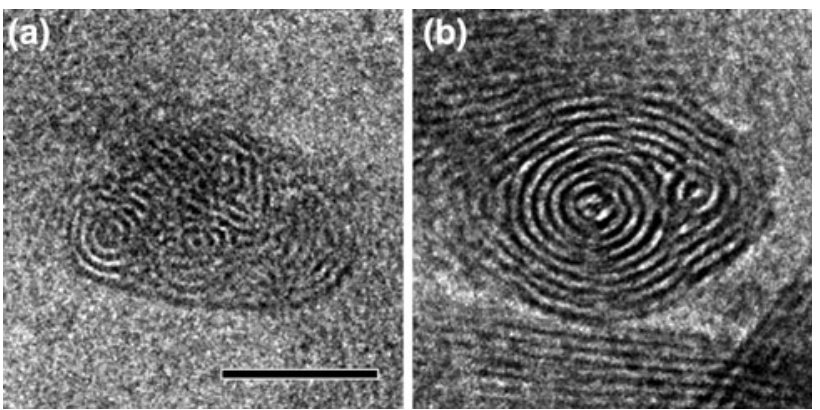

Fig. 3 End-on views of chlorosomes of Chlorobaculum tepidum, fixed in a vertical position in an amorphous ice layer. Cryo-EM reveals the packing of the lamellae. a Packing in the wild-type with some of the lamellae in concentric rings, others in a more irregular association. b Packing in the bchQRU mutant, showing a more regular multi-cylindrical organization. See also (Oostergetel et al. 2007) for further images. Size bar equals $25 \mathrm{~nm}$

stacking model are consistent with the intra-stack distance constraints derived from the NMR data (Ganapathy et al. 2009). When stacks are combined into sheets (Fig. 5b), several inter-stack distances in the antiparallel monomer stacking configuration are larger than those derived from the NMR measurements, whereas the syn-anti monomer stack assemblies are consistent with the observed distance constraints.

In chlorophyll aggregates, the ${ }^{1} \mathrm{H}$ NMR signals shift upfield by ring current effects from neighbouring molecules. Ring current shift calculations were performed for the synanti monomer stack, the antiparallel monomer model and two earlier structural models that were proposed for BChl $c$ in chlorosomes: the monomer-based parallel-stack model (Holzwarth and Schaffner 1994) and the piggy-back dimer model (Egawa et al. 1975). The calculated shifts for the antiparallel monomer stack and the piggy-back dimer configuration were much larger than the experimental shifts. Calculations on the syn-anti monomer stack and parallel stack reproduced the experimentally observed shifts. Since the parallel-stack model and the piggy-back dimer model did not satisfy the NMR distance constraints, it was concluded that the syn-anti monomer stack was the only model that was consistent with experimental NMR observations and theoretical calculations (Ganapathy et al. 2009).

Based on this syn-anti dimer, optimized by molecular mechanics calculations, and the cryo-EM observations, cylindrical models were constructed. For the bchQRU mutant, the strong $0.83-\mathrm{nm}$ periodicity in the direction of the long axis (Fig. 4c, d) can be explained by placing the BChl stacks along the circumference of co-axial cylinders, perpendicular to the cylinder axis (Fig. 5, 6). The stacks interconnect via hydrogen bonds which create ultrafast helical exciton delocalization pathways along the $\mathrm{BChl}$ cylinders. In the wild-type chlorosomes, the BChl stacks are oriented in the direction of the long axis. Again a 

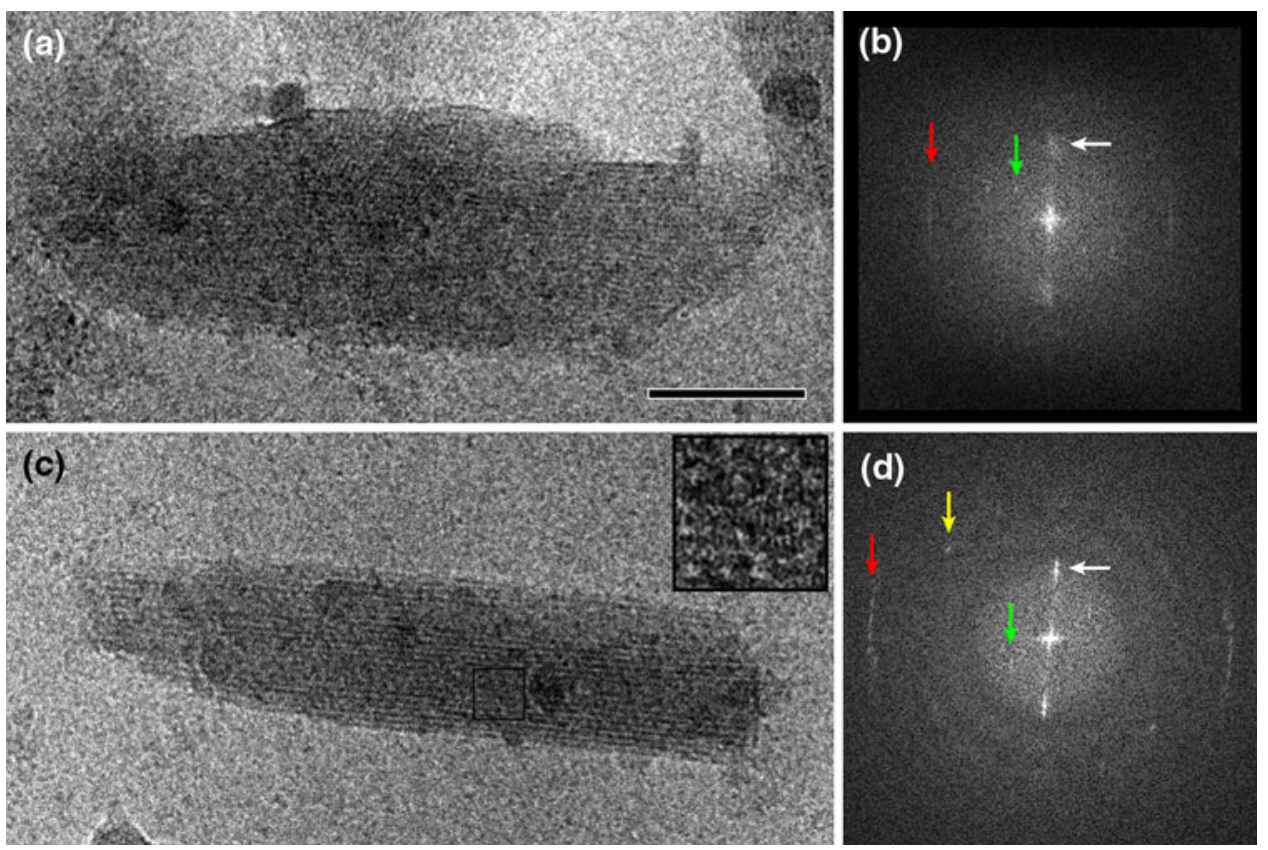

Fig. 4 Analysis of the interior of the chlorosome of Chlorobaculum tepidum. a Image of an unstained, ice-embedded chlorosome from the wild-type. $\mathbf{b}$ Calculated diffraction pattern from the image of frame a. A bright but unsharp reflection spot (white arrow) indicates an average spacing between lamellae of $2.1 \mathrm{~nm}$, which is also directly visible in the image of frame a. A sharp layer line at $1.25 \mathrm{~nm}$ (red arrow) indicates a specific internal repeating distance of $1.25 \mathrm{~nm}$ of the lamellae, caused by a specific packing of BChls. A thin but distinct reflection at $3.3 \mathrm{~nm}$ (green arrow) is assigned to a spacing of

helical $\mathrm{O}-\mathrm{H} \cdots \mathrm{O}=\mathrm{C}$ exciton delocalization pathway is present, with opposite handedness as compared to the bchQRU mutant. The observed spacing of $1.25 \mathrm{~nm}$ (Fig. 4a, b) in this configuration is directly related to the size of a syn-anti heterodimer, the basic repeating unit, in the direction of the stack. Simulated projection images from these nanotube models and Fourier analysis confirmed that the supramolecular models were consistent with the experimental data (Fig. 7)

\section{Organization of the baseplate}

The chlorosome baseplate was first described as a 2D paracrystalline structure by freeze-fracture electron microscopy (Staehelin et al. 1980). It may be a monolayer of polar lipids, like the chlorosome envelope. Besides polar lipids, chlorosomes also contain non-polar lipids (waxes) (Sørensen et al. 2008), but their location is completely unknown. About 10 different proteins are embedded in the base plate. Among these, the most abundant is the 59residue chlorosome protein $\mathrm{A}(\mathrm{CsmA})$. The structure of apo-CsmA from $C$. tepidum was determined using NMR spectroscopy (Østergaard Pedersen et al. 2008). Overall, protein molecules of the baseplate. c Image of an unstained, iceembedded chlorosome from the bclQRU mutant. d Calculated diffraction pattern from the image of $\mathbf{c}$. The white and green arrows indicate structural elements as in the pattern of frame $\mathbf{b}$. The sharp layer line (red arrow) now indicates a specific internal repeating distance of $0.83 \mathrm{~nm}$, instead of $1.25 \mathrm{~nm}$ as in the wild-type. The yellow arrow marks a sharp reflection that hints at another type of spacing $(1.1 \mathrm{~nm})$, likely in the baseplate. Size bar equals $50 \mathrm{~nm}$ for frames $\mathbf{a}$ and $\mathbf{c}$

the 59-residue CsmA is predominantly $\alpha$-helical in nature with a long helical domain extending from residue 6-36, containing a putative $\mathrm{BChl} a$ binding domain, and a short helix in the C-terminal part extending from residue 41-49. The long $\mathrm{N}$-terminal $\alpha$-helical stretch is considered to be immersed into the lipid monolayer confining the chlorosome, whereas the short C-terminal helix is protruding outwards, thus supposedly being available for interaction with the FMO antenna protein. CsmA is known to form stable oligomers in the chlorosome baseplate $(\mathrm{Li}$ et al. 2006). In order to assemble two $\mathrm{BChl} a$ molecules in close connection, it was proposed that in the intact baseplate of the C. tepidum chlorosomes, CsmA exists as dimers (Østergaard Pedersen et al. 2008), which subsequently can be organized into long rows resulting in the $2 \mathrm{D}$ crystalline superstructure of the baseplate as observed by freezefracture electron microscopy (Staehelin et al. 1980).

Cryo-EM images of ice-embedded chlorosomes show a large variation of their angular positions. In some specific angular orientation, a thicker line is visible as a kind of a string of beads (Fig. 4a). The strings are considered to be baseplate protein rows in superposition. A calculated diffraction pattern of the part of the chlorosome with the string indicates a repeating distance of $3.3 \mathrm{~nm}$ (Fig. 4b). 

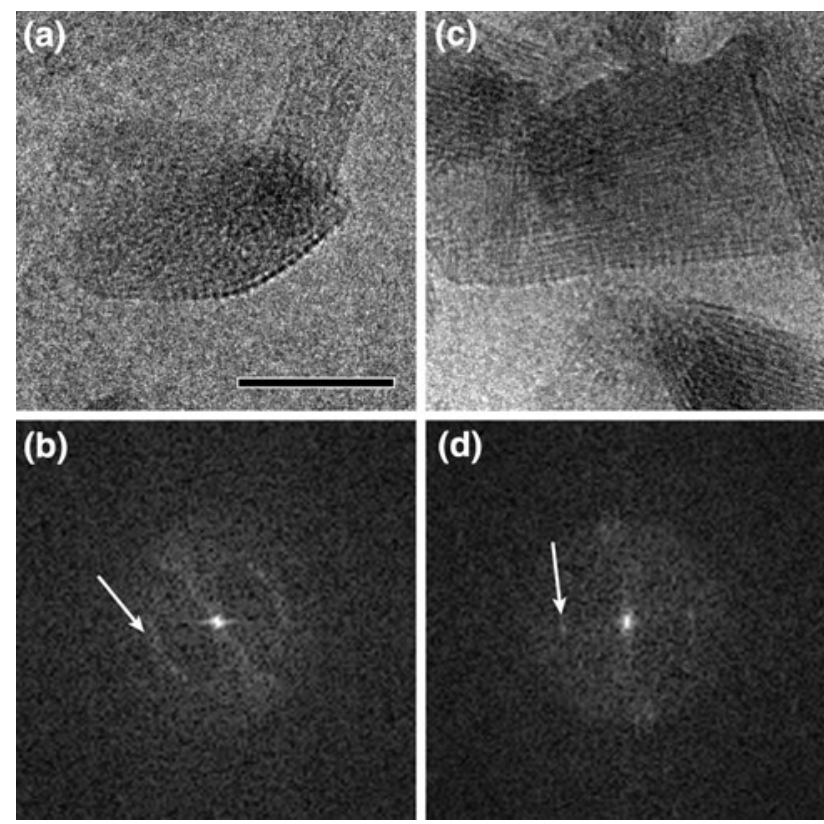

Fig. 5 Cryo-EM of Chlorobaculum tepidum chlorosomes. a A wildtype chlorosome recorded in an about vertical position (side view), and in a specific angular orientation in which rows of proteins of the baseplate become visible. b Diffraction pattern of a selected part of the chlorosome of frame a, showing that the elements at the edge have a repeating distance of $3.3 \mathrm{~nm}$ (white arrows). c A wild-type chlorosome in about horizontal position (top view). The baseplate element is not directly visible because of strong overlap with the interior. d Diffraction pattern of the chlorosome of frame $\mathbf{c}$, showing the same distance of $3.3 \mathrm{~nm}$ of elements as in frame b. G.T. Oostergetel, unpublished data). Size bar equals $50 \mathrm{~nm}$

The baseplates are not directly visible in chlorosomes in an about horizontal position, because the rows have strong overlap with the interior. (Fig. 4c). Diffraction, however, shows again the same distance of $3.3 \mathrm{~nm}$. The fact that the same spacing is observed in two positions is good evidence for the existence of a packing of CsmA molecules in rows with a width of $3.3 \mathrm{~nm}$. A dimer sandwich of CsmA plus BChl $a$ molecules would give such a width. A same conclusion was drawn from observed $3.3 \mathrm{~nm}$ spacings for the baseplate of Chloroflexus aurantiacus (Pšencík et al. 2009). The positions of spots in diffraction images indicate that the direction of the rows makes an angle of about $40^{\circ}$ with the long axis of the chlorosomes in C. tepidum but is approximately perpendicular to the long axis in $C f$. aurantiacus.

Other cryo-EM images hint at a smaller type of spacing, likely of the baseplate. A sharp reflection at $1.1 \mathrm{~nm}$ (yellow arrow, Fig. 4) must be caused by a smaller element of the baseplate. As $\alpha$-helices have about this dimension, they are the likely candidates. Pšenčík and colleagues observed a 0.8 -nm spacing in the direction of the long axis in their Xray scattering profiles (Pšenčík et al. 2009). Such spacing could be attributed to diffraction from the regular arrangement of CsmA protein in the baseplate as well,
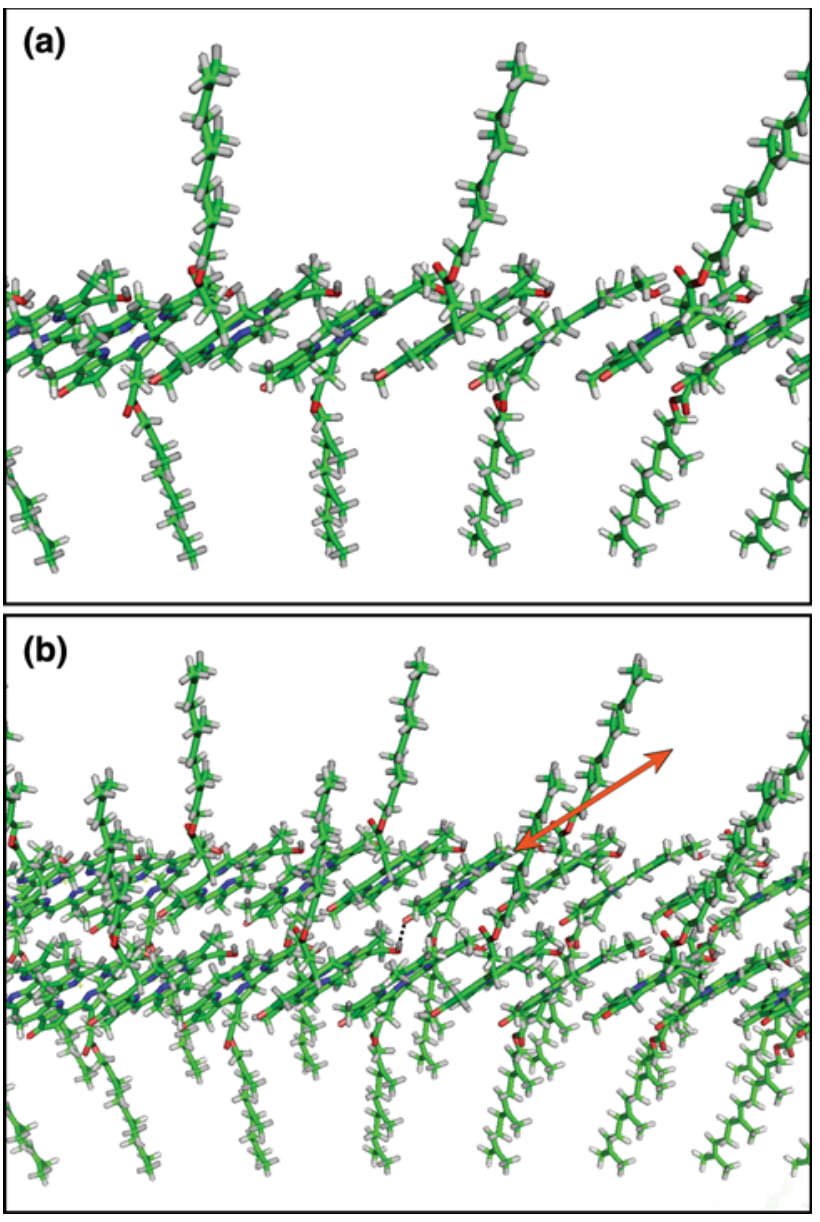

Fig. 6 Molecular models of BChl syn-anti monomer stacks in tubular models of a a single stack showing the farnesyl tails alternately extending on both sides. Radius of curvature $10.2 \mathrm{~nm}$. b Two syn-anti stacks interconnected by hydrogen bonds (black dotted line in the centre). The orange arrow indicates the direction of the exciton delocalization pathway over neighbouring stacks along the connecting hydrogen bonds. The models were made in Swiss-PDB Viewer and visualized using Pymol

although it seems to be too small to originate from a helical packing. Our recent cryo-EM observations do not confirm the 6-nm spacing observed by Staehelin et al. (1980), for which there is no logical explanation either.

\section{Light-harvesting and spectroscopic properties}

Spectroscopic properties in relation to function

Chlorosomes can contain hundreds of thousands of BChl $c$, $d$ or $e$ (depending on species), which are more closely related to chlorophylls than to bacteriochlorophylls (Blankenship and Matsuura 2003). Monomeric BChl $c$, for instance, has an absorption spectrum that is nearly identical to that of Chl $a$ with maxima around 436 and $668 \mathrm{~nm}$ in $\mathrm{CCl}_{4}$ (see, e.g. Olson and Pedersen 1990). Upon 


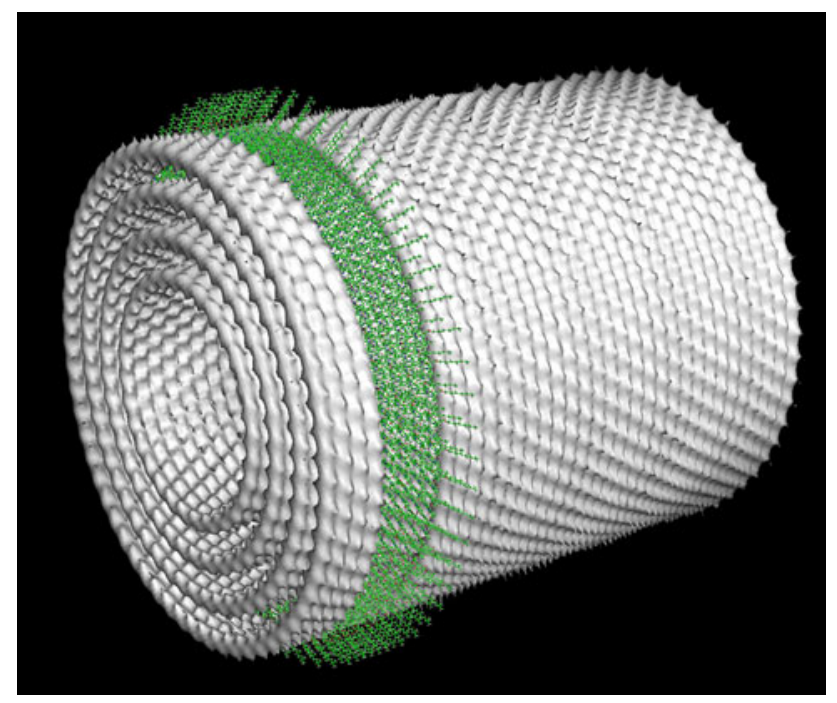

Fig. 7 Cylindrical model of the packing of concentric lamellae in the Chlorobaculum tepidum bchQRU mutant, based on distances as observed by electron microscopy and solid-state NMR spectroscopy (Ganapathy et al. 2009). The spacing between layers is $2.1 \mathrm{~nm}$. The green band indicates the position of individual Bchl molecules in four stacks of syn-anti dimers. In the wild-type chlorosomes, the stacks run in the direction of the cylinder axis

aggregation, the $\mathrm{BChl} c Q_{\mathrm{y}}$ absorption maximum shifts to $740-750 \mathrm{~nm}$, very similar to the position of the maximum observed in BChl $c$ containing chlorosomes and aggregates have often been studied as model systems for chlorosomes (see, e.g. Blankenship et al. 1995). Somewhat differently, the absorption maxima of chlorosomes that contain $\mathrm{BChl} d$ or $e$ are around 725 and $712 \mathrm{~nm}$, respectively (see, e.g. Blankenship and Matsuura 2003). The strong red shift upon aggregation is due to large excitonic interactions between BChl molecules that are partly stacked upon each other. Many studies have applied excitonic calculations to model and understand the spectroscopic properties of the chlorosomes (see, e.g. Lin et al. 1991; Martiskainen et al. 2009; Prokhorenko et al. 2003; Somsen et al. 1996) and the estimated coupling strengths between nearest-neighbour pigments typically range from -550 to $-750 \mathrm{~cm}^{-1}$. These large values lead to delocalization of the excitations over ten(s) of pigments (Prokhorenko et al. 2002; Savikhin et al. 1996, 1998) and they also allow excitations to travel extremely fast throughout the chlorosomes with a "transfer time" of tens of fs between neighbouring pigments as was, for instance, modelled (Prokhorenko et al. 2003). The excitation energy transfer (EET) throughout the chlorosome depends on the overall pigment organization which probably differs for different organisms. EET from bulk $\mathrm{BChl} c$ to baseplate $\mathrm{BChl} a$ in chlorosomes from $C f$. aurantiacus occurs for instance within $10 \mathrm{ps}$ (Martiskainen et al. 2009; Savikhin et al. 1996), while EET from bulk BChl $e$ to baseplate $\mathrm{BChl} a$ in chlorosomes from Chlorobium phaeobacteriodes is approximately 10 times as slow (Pšenčík et al. 2003).

The large coupling strengths are reminiscent of those in $\mathrm{J}$-aggregates but in that case they lead at the same time to substantial narrowing of the absorption bands (see, e.g. Fidder and Wiersma 1991). This is unfavourable for lightharvesting because this implies that only light in a very narrow wavelength region can be absorbed. However, the absorption bands of chlorosomes are rather broad which is at least partly due to the fact that the $\mathrm{BChl} c / d / e$ composition in vivo consists of a mixture of many homologues (Gomez Maqueo Chew et al. 2007; Olson and Pedersen 1990), which leads to structural disorder and thus to spectral broadening (see also (Prokhorenko et al. 2003 Somsen et al. 1996).

It is worthwhile to point out that the efficiency of EET to a RC is apart from the rate of EET and the number of pigments also determined by the ratio of the number of pigments in "contact" with the RC and the total amount of pigments. Suppose, for instance, that there would be 10 out of $10^{5}$ BChls in close contact to an RC $\left(N_{\text {transfer }}=10\right.$, $N_{\text {total }}=10^{5}$ ) and that the EET time from any of these 10 pigments to the $\mathrm{RC}$ would be $1 \mathrm{ps}$. Even if the energy transfer between the BChl $c$ molecules would be infinitely fast, the overall transfer time would be $N_{\text {total }} / N_{\text {transfer }}$ times $1 \mathrm{ps}=10 \mathrm{~ns}$, because the probability for excitations to be on a BChl $c$ next to the RC would be $N_{\text {transfer }} / N_{\text {total }}$, thereby lowering the effective transfer time to the $\mathrm{RC}$ with a factor of $10^{4}$ and also the transfer efficiency because of competing loss processes (fluorescence, internal conversion and intersystem crossing). The presence of a baseplate that contains BChl $a$ molecules which absorb at longer wavelengths (790-800 $\mathrm{nm}$ ) improves the efficiency of transfer to the RC (or in fact FMO protein/B808-866) substantially. Chlorosomes from Chloroflexaceae typically have a ratio BChl $c$ :BChl $a$ of 50 (Blankenship and Matsuura 2003), and the relatively large amount of BChl $a$ with excitedstate energy levels that are significantly below those of BChl $c$ leads to fast excited-state population within the baseplate $(\sim 10 \mathrm{ps}$, see also above). Transfer from baseplate to $\mathrm{RC}$ is a factor of $\sim 50$ faster than it would have been from BChl $c$ purely for entropic reasons because

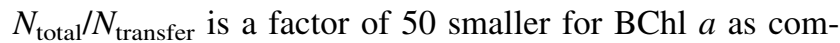
pared to BChl $c$. Of course, this is a simplified view because also other factors play a role like overlap of donor emission and acceptor absorption spectra and relative orientations of the transition dipole moments. By increasing the number of BChl $a$ molecules in the baseplate, the rate of extracting excitations from the $\mathrm{BChl} c$ pool will increase (also for entropic reasons) but on the other hand it will decrease the transfer to the RC because of lowering the ratio $N_{\text {total }} / N_{\text {transfer }}$ It is clear that the ratio of $\mathrm{BChl} c$ to Bchl $a$ is an important parameter for determining the 
efficiency of EET towards the RC but as far as we know no systematic research has been reported on this issue. In this respect, it might be interesting to note that for Chlorobiaceae the $\mathrm{BChl} c$ to $\mathrm{Bchl} a$ ratio is a factor of 10 higher, i.e. it is around 500 (Blankenship and Matsuura 2003).

The third category of pigments in chlorosomes is the one of the carotenoids, constituting $\sim 8 \%$ of the total amount of pigments in chloroflexaceae and $\sim 4 \%$ in chlorobiaceae (Blankenship and Matsuura 2003). They transfer excitation energy to the BChls and, for instance, in Cf. aurantiacus a transfer efficiency to BChl $c$ of $65 \%$ was reported (Van Dorssen et al. 1986), implying that at least $65 \%$ of the carotenoids should be in Van der Waals contact with BChl $c$. Direct interactions between BChls and carotenoids have also been inferred from changes in the BChl Stark spectrum (Frese et al. 1997) and the BChl absorption spectrum in the absence of carotenoids (Arellano et al. 2000; Kim et al. 2007). On the other hand, the carotenoids also protect chlorosomes against photodegradation and it was found that carotenoid-free chlorosomes photodegrade approximately three times faster than wild-type ones (Kim et al. 2007). However, no proof for BChl $c$ triplet quenching by carotenoids could be found in $C f$. aurantiacus and $C$. tepidum (Carbonera et al. 2001), whereas Arellano and coworkers found evidence for BChl $a$ triplet quenching by carotenoids but not for BChl $e$ triplet quenching in Chlorobium phaeobacteroides strain CL1401 (Arellano et al. 2000). Triplet quenching of (B)Chls by nearby carotenoids is usually occurring in photosynthetic light-harvesting systems to avoid the formation of deleterious singlet oxygen. It was demonstrated that monomeric $\mathrm{BChl} e$ sensitizes singlet oxygen formation but aggregated $\mathrm{BChl} e$ does not (Arellano et al. 2002). In line with these results, Kim and colleagues studied a carotenoid-free mutant of BChl $c$ containing C. tepidum and found that a significant fraction of the BChls forms a long-lived, triplet-like state that does not interact with oxygen and it was proposed that these states are triplet excitons formed by triplet-triplet interaction between BChls that are lower in energy than the singlet oxygen state (but also than the triplet energy level of carotenoids) (Kim et al. 2007).

\section{Light spectroscopy and structure}

The large excitonic red shift of the chlorosomes requires an arrangement of the pigments that is reminiscent of the organization in J-aggregates (Moll et al. 1995), i.e. head-totail or head-to-head organization and many possibilities have been provided in literature over the years (for an "early" overview see, for instance, Blankenship et al. 1995). Most of these proposed aggregates were linear but to account for the relatively pronounced circular dichroism (CD) helical and cylindrical models were introduced (Lin et al. 1991; Prokhorenko et al. 2003; Somsen et al. 1996; Linnanto and Korppi-Tommola 2008) in which the J-type organization was kept intact. Over the years also many linear-dichroism (LD) measurements have been performed and these all demonstrated that the transition dipole moment corresponding to the long-wavelength $Q_{\mathrm{y}}$ transition dipoles make a relatively small angle with the long axis of the chlorosomes (for more details see below). Also polarized transient absorption measurements (Lin et al. 1991; Pšenčík et al. 2003) and polarized fluorescence measurements on non-oriented chlorosomes (Ma et al. 1996; Van Dorssen et al. 1986) and chlorosomes in intact cells of $C$. limicola (Fetisova et al. 1988) indicated a high degree of ordering, that was more or less consistent with the $\mathrm{LD}$ results.

As LD measurements provide spectroscopic information that may be used to verify structural models we will briefly address the LD of chlorosomes. The LD $(\Delta A)$ is defined as the difference in absorption $(A)$ of light polarized parallel $(v)$ and perpendicular $(h)$ to the orientation axis of the sample (expansion direction of a squeezed gel containing the chlorosomes or the direction of an orienting electric field): $\Delta A=A_{v}-A_{h}$ (see also Garab and Van Amerongen 2009). LD measurements provide the angle $\theta$ between a transition dipole moment and the long axis of the chromosome. Values between $15^{\circ}$ and $27^{\circ}$ were obtained for the transition dipole moment of the main $Q_{\mathrm{y}}$ band and the long axis of the chlorosomes from Cf. aurantiacus (Frese et al. 1997; Griebenow et al. 1991; Matsuura et al. 1993; Van Amerongen et al. 1988, Van Amerongen et al. 1991). Single molecule experiments on chlorosomes from $C f$. aurantiacus also showed preferential orientation of the $Q_{\text {y }}$ dipole moment along the long axis, and from these results an average angle of around $29^{\circ}$ can be inferred. Recent experiments on chlorosomes from $C$. limicola are somewhat different but also show a high degree of ordering (Shibata et al. 2009).

It should be noted that the orientation of the main $Q_{\mathrm{y}}$ transition dipole is not necessarily parallel to the transition dipole moment of the individual BChls. For ideal helical and cylindrical models in which the broadening of the absorption bands is ignored, the red-most band is parallel to the helix/cylinder axis (positive LD), whereas degenerate perpendicular components absorb more to the blue (Lin et al. 1991; Somsen et al. 1996), creating negative LD. However, when homogeneous and inhomogeneous broadenings of the absorption bands are also included, the picture is less extreme and the reduced LD decreases more gradually upon going to the blue. Such a decrease has indeed been reported (Griebenow et al. 1991; Matsuura et al. 1993). Earlier polarized transient absorption measurements showed a decrease in anisotropy upon going to the blue and it was explained in a similar way (Lin et al. 1991). 
Although the angle reported above refers to the transition dipole moments of excitonic transitions, the orientations of the transition dipole moments of the individual pigments can be obtained in a straightforward way. In fact, if one integrates the LD over the entire $Q_{\mathrm{y}}$ band and compares it to the integrated absorption, one obtains the angle of the transition dipole moment of the individual BChls with respect to the long axis of the chlorosomes (for the background theory we refer to (Somsen et al. 1996; Van Amerongen et al. 2000), but the underlying reason is that excitonic interactions shift absorption bands but do not alter the total amount of dipole strength along a particular axis). Although it has never been explicitly calculated in literature, it can easily be done from the available data and it appears that the obtained angle for the individual pigments is at most a few degrees larger than the one of the main (excitonic) absorption band. Thus, it is concluded that the above-mentioned results on chlorosomes from $C f$. $a u$ rantiacus demonstrate that the angle between the $Q_{\mathrm{y}}$ transition dipole moment of the individual $\mathrm{BChl} c$ molecules is $25^{\circ} \pm 6^{\circ}$ with respect to the long axis, where the error reflects the spread in the reported values. These numbers can be taken into account when building molecular models (Prokhorenko et al. 2003).

There is a remarkable variability in the shape of the CD spectra that have been reported in literature. This variability was even present for chlorosomes that were prepared in an identical way, whereas the absorption and linear-dichroism spectra were identical. It was demonstrated in (Somsen et al. 1996) that a slight reorganization of cylindrical aggregates could explain these results, but later it was demonstrated that the variability in CD could elegantly be explained by variations in the length of the cylindrical aggregates (which do not substantially affect the absorption and LD spectra (Didraga et al. 2002; Didraga and Knoester 2003; Prokhorenko et al. 2003).

In the recently proposed model for chlorosomes from a triple mutant of $C$. tepidum, the $Y$-axis of BChl $c$ along which the $Q_{\mathrm{y}}$ transition dipole moment is oriented makes an angle of $55^{\circ}$ with the local cylinder axis (Ganapathy et al. 2009). This means that the LD integrated over the $Q_{\mathrm{y}}$ band should be very close to zero. Due to exciton coupling, the LD is again expected to be positive on the longwavelength side and to keep the integrated LD close to zero this should then be compensated by negative LD on the short-wavelength side. Linear-dichroism spectra of these particular chlorosomes have not been presented in literature.

There is one more issue that should be clarified and this concerns the Stark spectrum of chlorosomes. Chls and BChls possess a difference dipole moment $\Delta \mu$ between ground and excited $\left(Q_{\mathrm{y}}\right)$ state that is responsible for a feature in the Stark spectrum with the shape of the second- derivative of the absorption spectrum (see, e.g. Boxer, 2009). The intensity of this contribution is a measure for the value of $\Delta \mu$. Remarkably, in contrast to all the known Stark spectra of photosynthetic complexes, there is no such feature for the $Q_{\mathrm{y}}$ absorption band and $\Delta \mu$ is equal to 0 (Frese et al. 1997). This has been explained by an antiparallel organization of strongly coupled BChl $c$ molecules in the chlorosome, either because of antiparallel-dimer building blocks or because of the presence of antiparallel linear stacks. Such an antiparallel organization is not present in the model for the chlorosomes of triple mutant of C. tepidum mentioned above (Ganapathy et al. 2009). Therefore, it is expected that Stark measurements on these chlorosomes will show second-derivative character in the $Q_{\text {y }}$ region and together with the LD measurements they might form another way of testing the current model. Finally, it is worthwhile to point out that the lamellar model that was proposed by Pšenčík et al. (2004) cannot explain the pronounced CD spectra of chlorosomes (Linnanto and Korppi-Tommola 2008) although the authors could not rule out the simultaneous presence of lamellar and cylindrical structures. According to the most recent EM data presented above, such a coexistence seems indeed to be the case (Ganapathy et al. 2009; Oostergetel et al. 2007).

\section{Closing remarks}

In conclusion, chlorosomes are fascinating organelles because of their amazing capacity of light harvesting. The need for harvesting a broad range of the spectrum of light constrains the composition of the BChl molecules and the amount of order in the packing, for which now a consistent model is available. This model describes the molecular and supramolecular packing and can be further tested, for instance, with LD, which will provide useful information on the long-range ordering of the pigments. An intriguing feature is the thin envelope which consists of only one membrane leaflet, an uncommon phenomenon in nature. It nevertheless contains 10 different proteins, and some of them play important roles in determining chlorosome size as well as the assembly and supramolecular organization of the BChl $c$ aggregates within the chlorosome (Li and Bryant 2009). It shows the complicated fine tuning of the participating components. As the determined interactions between the BChls, however, are rather simple it may one day be possible to build artificial photosynthetic chlorosome-based systems that efficiently convert solar energy to electricity or fuel.

Acknowledgements We thank Dr. Don Bryant for providing us with a sample of Cab. thermophilum. Work has been supported by the 
Counsel for Chemical Research of the Netherlands Organization for Scientific Research (NWO).

Open Access This article is distributed under the terms of the Creative Commons Attribution Noncommercial License which permits any noncommercial use, distribution, and reproduction in any medium, provided the original author(s) and source are credited.

\section{References}

Arellano JB, Melo TB, Borrego CM, Garcia-Gil J, Naqvi KR (2000) Nanosecond laser photolysis studies of chlorosomes and artificial aggregates containing bacteriochlorophyll e: Evidence for the proximity of carotenoids and bacteriochlorophyll a in chlorosomes from Chlorobium phaeobacteroides strain CL1401. Photochem Photobiol 72:669-675

Arellano JB, Melo TB, Borrego CM, Naqvi KR (2002) Bacteriochlorophyll e monomers, but not aggregates, sensitize singlet oxygen: implications for a self-photoprotection mechanism in chlorosomes. Photochem Photobiol 76:373-380

Balaban TS, Tamiaki H, Holzwarth AR (2005) Chlorins programmed for self-assembly. Top Curr Chem 258:1-38

Blankenship RE, Matsuura K (2003) Antenna complexes from green photosynthetic bacteria. In: Green BR, Parson WW (eds) Lightharvesting antennas in photosynthesis. Kluwer Academic Publishers, Dordrecht, pp 195-217

Blankenship RE, Olson JM, Miller M (1995) Antenna complexes from green photosynthetic bacteria. In: Blankenship RE, Madigan MT, Bauer CE (eds) Anoxygenic photosynthetic bacteria. Kluwer Academic Publishers, Dordrecht, pp 399-435

Boxer SG (2009) Stark realities. J Phys Chem B 113:2972-2983

Bryant DA, Frigaard NU (2006) Prokaryotic photosynthesis and phototrophy illuminated. Trends Microbiol 14:488-496

Bryant DA, Costas AMG, Maresca JA, Chew AGM, Klatt CG, Bateson MM, Tallon LJ, Hostetler J, Nelson WC, Heidelberg JF, Ward DM (2007) Candidatus Chloracidobacterium thermophilum: an aerobic phototrophic acidobacterium. Science 317:523-526

Carbonera D, Bordignon E, Giacometti G, Agostini G, Vianelli A, Vannini C (2001) Fluorescence and absorption detected magnetic resonance of chlorosomes from green bacteria Chlorobium tepidum and Chloroflexus aurantiacus. A comparative study. J Phys Chem B 105:246-255

Cohen-Bazire G, Pfennig N, Kunisawa R (1964) Fine structure of green bacteria. J Cell Biol 22:207-225

Didraga C, Knoester J (2003) Absorption and dichroism spectra of cylindrical $\mathrm{J}$ aggregates and chlorosomes of green bacteria. J Lumin 102:60-66

Didraga C, Klugkist JA, Knoester J (2002) Optical properties of helical cylindrical molecular aggregates: the homogeneous limit. J Phys Chem 106:11474-11486

Egawa A, Fujiwara T, Mizoguchi M, Kakitani Y, Koyama Y, Akutsu $\mathrm{H}$ (1975) Structure of the light-harvesting bacteriochlorophyll c assembly in chlorosomes from Chlorobium limicola determined by solid-state NMR. Proc Natl Acad Sci USA 104:790-795

Fenna RE, Matthews BW (1975) Chlorophyll arrangement in a bacteriochlorophyll protein from Chlorobium limicola. Nature 258:573-577

Fetisova ZG, Freiberg AM, Timpmann KE (1988) Long-range molecular order as an efficient strategy for light-harvesting in photosynthesis. Nature 334:633-634

Fidder H, Wiersma DA (1991) Resonance light-scattering study and line-shape simulation of the J-band. Phys Rev Lett 66:15011504
Frese R, Oberheide U, van Stokkum IHM, van Grondelle R, Foidl M, Oelze J, van Amerongen H (1997) The organization of bacteriochlorophyll $c$ in chlorosomes from Chloroflexus aurantiacus and the structural role of carotenoids and protein-an absorption, linear dichroism, circular dichroism and Stark spectroscopy study. Photosynth Res 54:115-126

Ganapathy S, Oostergetel GT, Wawrzyniak PK, Reus M, Gomez Maqueo Chew A, Buda F, Boekema EJ, Bryant DA, Holzwarth AR, de Groot HJM (2009) Alternating syn-anti bacteriochlorophylls form concentric helical nanotubes in chlorosomes. Proc Natl Acad Sci USA 106:8525-8530

Garab G, Van Amerongen H (2009) Linear dichroism and circular dichroism in photosynthesis research. Photosynth Res 101: 135-146

Gomez Maqueo Chew A, Frigaard NU, Bryant DA (2007) Bacteriochlorophyllide $c$ C-8(2) and C-12(1) Methyltransferases are essential for adaptation to low light in Chlorobaculum tepidum. J Bacteriol 189:6176-6184

Griebenow K, Holzwarth AR, van Mourik F, van Grondelle R (1991) Pigment organization and energy transfer in green bacteria. 2 . Circular and linear dichroism spectra of protein-containing and protein-free chlorosomes isolated from Chloroflexus aurantiacus strain OK-70-ff*. Biochim Biophys Acta 1058:194-202

Hauska G, Schoedle T, Remigy H, Tsiotis G (2001) The reaction center of green sulfur bacteria. Biochim Biophys Acta 1507: 260-277

Higuchi M, Hiramo Y, Kimura Y, Oh-oka H, Miki K, Wang ZY (2009) Overexpression, characterization, and crystallization of the functional domain of cytochrome $\mathrm{cz}$ from Chlorobium tepidum. Photosynth Res 102:77-84

Holzwarth AR, Schaffner K (1994) On the structure of bacteriochlorophyll molecular aggregates in the chlorosomes of green bacteria-a molecular modeling study. Photosynth Res 41: 225-233

Kim H, Li H, Maresca JA, Bryant DA, Savikhin S (2007) Triplet exciton formation as a novel photoprotection mechanism in chlorosomes of Chlorobium tepidum. Biophys J 93:192-201

Li H, Bryant D (2009) Envelope proteins of the CsmB/CsmF and CsmC/CsmD motif families influence the size, shape, and composition of chlorosomes in Chlorobaculum tepidum. J Bacteriol 191:7109-7120

Li YF, Zhou W, Blankenship RE, Allen JP (1997) Crystal structure of the of the bacteriochlorophyll $a$ protein from Chlorobium tepidum. J Mol Biol 271:456-471

Li H, Frigaard NU, Bryant D (2006) Moleculear contacts for chlorosome proteins revealed by cross-linking studies with clorosomes from Chlorobium tepidum. Biochemistry 45:90959103

Lin S, Van Amerongen H, Struve WS (1991) Ultrafast pump-probe spectroscopy of bacteriochlorophyll $\mathrm{c}$ antennae in bacteriochlorophyll a-containing chlorosomes from the green photosynthetic bacterium Chloroflexus aurantiacus. Biochim Biophys Acta 1060:13-24

Linnanto JM, Korppi-Tommola JEI (2008) Investigation on chlorosomal antenna geometries: tube, lamella and spiral-type selfaggregates. Photosynth Res 96:227-245

Ma YZ, Cox RP, Gillbro T, Miller M (1996) Bacteriochlorophyll organization and energy transfer kinetics in chlorosomes from Chloroflexus aurantiacus depend on the light regime during growth. Photosynth Res 47:157-165

Manske AK, Glaeser J, Kuypers MAM, Overmann J (2005) Physiology and phylogeny of green sulfur bacteria forming a monospecific phototrophic assemblage at a depth of 100 meters in the Black Sea. Appl Environ Microb 71:8049-8060

Martiskainen J, Linnanto J, Kananavicius R, Linnanto JM, Kananavičius R, Lehtovuori V, Korppi-Tommola J (2009) Excitation 
energy transfer in isolated chlorosomes from Chloroflexus aurantiacus. Chem Phys Lett 477:216-220

Matsuura K, Hirota M, Shimada K, Mimuro M (1993) Spectral forms and orientation of bacteriochlorophyll- $c$ and bacteriochlorophyll$a$ in chlorosomes of the green photosynthetic bacterium Chloroflexus aurantiacus. Photochem Photobiol 57:92-97

Moll J, Daehne S, Durrant JR, Wiersma DA (1995) Optical dynamics of excitons in J-aggregates of a carbocyanine dye. J Chem Phys 102:6362-6370

Oh-oka H (2007) Type 1 reaction center of photosynthetic heliobacteria. Photochem Photobiol 83:177-186

Olson JM, Pedersen JP (1990) Bacteriochlorophyll-c monomers, dimers, and higher aggregates in dichlormethane, chloroform, and carbon-tetrachloride. Photosynth Res 25:25-37

Oostergetel GT, Reus M, Gomez Maqueo Chew A, Bryant DA, Boekema EJ, Holzwarth AR (2007) Long-range organization of bacteriochlorophyll in chlorosomes of Chlorobium tepidum investigated by cryo-electron microscopy. FEBS Lett 581:5435-5439

Østergaard Pedersen M, Underhaug J, Dittmer J, Miller M, Nielsen NC (2008) The three-dimensional structure of CsmA: a small antenna protein from the green sulfur bacterium Chlorobium tepidum. FEBS Lett 582:2869-2874

Prokhorenko VI, Holzwarth AR, Muller MG, Schaffener K, Miyatake T, Tamiaki H (2002) Energy transfer in supramolecular artificial antennae units of synthetic zinc chlorins and coaggregated energy traps: a time-resolved fluorescence study. J Phys Chem B 106:5761-5768

Prokhorenko VI, Steensgaard DB, Holzwarth AR (2003) Exciton theory for supramolecular chlorosomal aggregates. 1. Aggregate size dependence of the linear spectra. Biophys J 85:3173-3186

Pšenčík J, Ma YZ, Arellano JB, Hala J, Gillbro T (2003) Excitation energy transfer dynamics and excited-state structure in chlorosomes of Chlorobium phaeobacteroides. Biophys J 84:1161-1179

Pšenčík J, Ikonen TP, Laurinmäki P, Merckel MC, Butcher SJ, Serimaa RE, Tuma R (2004) Lamellar organization of pigments in chlorosomes, the light harvesting complexes of green photosynthetic bacteria. Biophys J 87:1165-1172

Pšenčík J, Collins AM, Liljeroos L, Torkkeli M, Laurinmaki P, Ansink HM, Ikonen TP, Serimaa RE, Blankenship RE, Tuma R, Butcher SJ (2009) Structure of chlorosomes from the green filamentous bacterium Chloroflexus aurantiacus. J Bacteriol 191:6701-6708

Savikhin S, Zhu YW, Blankenship R, Stuve WS (1996) Ultrafast energy transfer in chlorosomes from the green photosynthetic bacterium Chloroflexus aurantiacus. J Phys Chem 100:33203322

Savikhin S, Buck DR, Struve WS, Blankenship R, Taisova AS, Novoderezhkin VI, Fetisova ZG (1998) Excitation delocalization in the bacteriochlorophyll $\mathrm{c}$ antenna of the green bacterium Chloroflexus aurantiacus as revealed by ultrafast pump-probe spectroscopy. FEBS Lett 430:323-326

Shibata Y, Saga Y, Tamiaki H, Itoh S (2009) Anisotropic distribution of emitting transition dipoles in chlorosome from Chlorobium tepidum: fluorescence polarization anisotropy study of single chlorosomes. Photosynth Res 100:67-78

Somsen OJG, van Grondelle R, van Amerongen H (1996) Spectral broadening of interacting pigments: polarized absorption by photosynthetic proteins. Biophys J 71:1934-1951

Sørensen PG, Cox RP, Miller M (2008) Chlorosome lipids from Chlorobium tepidum: characterization and quantification of polar lipids and wax esters. Photosynth Res 95:191-196

Staehelin LA, Golecki JR, Fuller RC, Drews G (1978) Visualization of the supramolecular architecture of chlorosomes (Chlorobium type vesicles) in freeze-fractured cells of Chloroflexus-Aurantiacus. Arch Microbiol 119:269-277

Staehelin LA, Golecki JR, Drews G (1980) Supramolecular organization of chlorosomes (Chlorobium vesicles) and of their membrane attachment sites in Chlorbium limicola. Biochim Biophys Acta 589:30-45

Tronrud DE, Schmid MF, Matthews BW (1986) Structure and X-ray amino acid sequence of a bacteriochlorophyll $a$ protein from Prosthecochloris aestuarii refined at $1.9 \AA$ resolution. J Mol Biol 188:443-454

Tronrud DE, Wen JZ, Gay L, Blankenship RE (2009) The structural basis for the difference in absorbance spectra for the FMO antenna protein from various green sulfur bacteria. Photosynth Res 100:79-87

Van Amerongen H, Vasmel H, van Grondelle R (1988) Linear dichroism of chlorosomes from Chloroflexus aurantiacus in compressed gels and electric fields. Biophys J 54:65-76

Van Amerongen H, Van Haeringen B, Van Gurp M, Van Grondelle R (1991) Polarized fluorescence measurements on ordered photosynthetic antenna complexes-chlorosomes of Chloroflexus aurantiacus and B800-B850 antenna complexes of Rhodobacter sphaeroides. Biophys J 59:992-1001

Van Amerongen H, Valkunas L, van Grondelle R (2000) Photosynthetic excitons. World Scientific, Singapore, ISBN 981-02-3280-2

Van Dorssen RJ, Vasmel H, Amesz J (1986) Pigment organization and energy-transfer in the green photosynthetic bacterium Chloroflexus aurantiacus. 2. The chlorosome. Photosynth Res 9:33-45

Wen J, Zhang H, Gross ML, Blankenship RE (2008) Membrane orientation of the FMO antenna protein from Chlorobaculum tepidum as determined by mass spectrometry-based footprinting. Proc Natl Acad Sci USA 106:6134-6139 